\title{
Status of Water, Hygiene and Sanitation Practices in Southern Rwanda
}

\author{
Theoneste Ntakirutimana $^{1}$, Malachie Tuyizere ${ }^{1 *}$, Olivier Ndizeye ${ }^{2}$, Francois Xavier Sunday ${ }^{1}$ \\ ${ }^{1}$ College of Medicine and Health Sciences, University of Rwanda, Kigali, Rwanda \\ ${ }^{2}$ WaterAid Rwanda
}

*Corresponding author: Malachie Tuyizere. College of Medicine and Health Sciences, University of Rwanda, Remera Campus, KG 11 Ave, 47, Kigali, Rwanda. Email: malaschie@gmail.com

\begin{abstract}
Background

Increasing access to water sanitation and promoting basic hygiene behaviours can reduce the burden of diarrheal diseases. Availability of clean water and soap enables and encourages people to wash their hands, and as a result, it reduces the likelihood of disease transmission. The study intended to assess the hygiene and sanitation practices in Southern Rwanda.
\end{abstract}

\section{Methods}

A mixed method with quantitative and qualitative approach was used. A random sample of 291 households was included in the study. Focus Group Discussions (FGDs), Key Informant Interviews (KIIs), and observations were used. The data was analysed using SPSS 21.

\section{Results}

The findings show that $88 \%$ of respondents had knowledge on best practices of hand washing with soap; $83.5 \%$ of the respondents own latrines, and $38 \%$ and $26 \%$ had the will to improve their toilets roof and slabs respectively.

Forty-four per cent of respondents use boiling water methods and 55\% do not treat water at all. Boiling water was regarded as the main water treatment method.

\section{Conclusion}

The study concludes that lack of water and soaps, and hand washing facilities were among other factors that hinder hygiene and sanitation.

Key words: Hygiene; sanitation practice

\section{INTRODUCTION}

Globally, limited access to water and low level of sanitation and hygiene practice are responsible for $90 \%$ of diarrhoea-related mortality.[1] Increasing access to water, sanitation and promoting adequate hygiene behaviour can reduce the burden of diarrheal diseases. Availability of clean water and soap enables and encourages people to wash their hands especially at critical times, and as a result, it reduces the likelihood of disease transmission. Good and safe sanitation can control flies and other insects that spread disease, and prevent contact with infectious organisms shed in faeces. This also prevents contamination of food or ustensils. Drinking safe water can prevent the spread of waterborne diseases among the population.[2]

In September 2015, the 2030 Agenda for Sustainable Development Goals (SDGs) was adopted by world leaders at the UN General Assembly, which calls upon countries to begin new efforts to achieve 17 SDGs over the next 15 years, including the goal to ensure the availability and sustainable management of water and sanitation 
and hygiene for all. The provision of water sanitation and hygiene is an important intervention within programs aiming at the prevention and provision of healthcare for all neglected tropical diseases.[3] In Rwanda, the water and sanitation policies and strategies approved in 2016 provided a framework for achieving universal access to WASH services. Rwanda is ambitious to achieve $100 \%$ access to safely managed water and sanitation services by the year 2030.[4] However, there are still some challenges including the funding gaps to increasing access to WASH services, particularly in scattered settlements in rural areas. [5-7] Moreover, the scale of the need for safe water, sanitation and hygiene; and how best to sustain WASH services and to reach people most in need; and the integration of water, sanitation and hygiene were all well highlighted in the literature. [8]

In the effort to address WASH issues, the Government of Rwanda with support from UNICEF and the Government of the Netherlands, implemented a six-year WASH project from 2009. The Government of Rwanda has implemented the Community-Based Environmental Health Promotion Program (CBEHPP). The CBEHPP is a program consisting in identifying personal and domestic hygiene and environmental related problems. The CBEHPP model has proved to be an efficient tool to rapidly solve the problems in the area of WASH. In this model, the engagement of the Community Hygiene Club (CHC) members has unveiled the full potential for the communities to solve their WASH problems, and since then, much effort was driven to strengthening $\mathrm{CHCs}$ and increasing their skills to solve the community WASH problems in their respective villages. Despite the existing efforts to address WASH problems, inadequate and unhygienic facilities for excreta disposal, poor management of waste, and poor hand washing practices still contribute to an estimated $88 \%$ of diarrheal diseases. Inadequate WASH can cause various adverse health outcomes, through a number of different transmission pathways including lack of water linked to inadequate personal hygiene, contact with contaminated water, and others.[9]

The current formative survey therefore sought to gather evidence on current WASH practices and possible factors that influence them, in Nyamagabe district in Southern Rwanda. In addition, the study aimed at identifying the potential touch points to reach people during implementation of intervention to address challenges regarding hygiene and sanitation practices in the study area but this will not be discussed in this paper.

\section{METHODS}

\section{Study design and population}

This survey was conducted through a crosssectional design using both quantitative and qualitative approaches. The study targeted a population of 85,478 households distributed into 17 sectors and 536 villages in Nyamagabe district, in 2019.

\section{Sample size and sampling strategy}

A two-stage sampling method was used to select a representative sample for this survey. In the first stage, all 17 sectors were included. In the second stage, a simple random sampling with Excel random generator was used to select a representative sample from each village. The household representative or in his absence, another person at least aged 18 years old was eligible for household interview. The probabilistic sample size calculation and selection was done using the formula:

$$
\mathrm{ME}=z \sqrt{\frac{\hat{p}(1-\hat{p})}{n}}
$$

Where:

ME is the desired margin of error (0.05)

$\mathbf{Z}$ is the $z$-score (1.96)

p is the proportion of households with improved sanitation which is $87 \%$ according to EICV5 (The Fifth Integrated Household Living Conditions Survey or Enquête Intégrale sur les Conditions de Vie des ménages in French).

$\mathbf{n}$ is the sample size

We considered the precision of $5 \%$, a $95 \%(Z 1-\infty / 2)$ with confidence level of 1.96 and $\mathrm{p}$ of 0.87 (the proportion of households with improved sanitation of $87 \%$ according to EICV5 [10]). The final sample size was 291 households (including a minimum 10\% non-response rate). 14 villages were selected by the method of 1000 households per 50 locations referred to as clusters (villages). Ten (10) Focus Group discussions (FGDs) were conducted with Community Hygiene Club members, and heads of households with at least one under five child. The FGDs were determined from five geographical 
locations (North, South, West, East, Centre) with two FGDs from each. In addition, thirteen (13) Key Informant Interviews (KIIs) were conducted with local leaders.

\section{Data collection tools}

Quantitative data were collected using questionnaire for households and questionnaire for village leaders that were pretested for clarity or existence of any possible gaps, flow of the questions and time required to complete the interview. Practical problems identified were considered for questionnaire validation. All questionnaires were translated into Kinyarwanda, the local language for all participants. Quantitative data were collected using tablets with installed mWater surveyor software. Focus Group discussions and Key informant interview guides were used for qualitative data collection. Recorders were used to register the data from participants.

\section{Data analysis}

The data were downloaded from mWater surveyor software into Excel sheet for analysis. Quantitative data was analysed using SPSS 21, and respondents' behavioural patterns were quoted for further understanding of quantitative findings.

\section{Ethical Considerations}

The survey was approved by the UR-CMHS Institutional Review Board (IRB) and Nyamagabe district authority granted permission. Signed consents were also obtained from participants before starting the interviews.

\section{RESULTS AND DISCUSSION}

This section summarizes and discusses the main findings from this baseline assessment of hygiene and sanitation conducted in Southern Rwanda. The assessment focused on hand washing with soap and food hygiene practices as well as household water treatment and storage and faecal management.

\section{Sociodemographic participants ( $n=291)$}

characteristics

of

Gender analysis of the participants shows that 70.8 $\%$ were males while $29.2 \%$ were females; males participated more compared to females because the study targeted heads of households who are usually males.[11] The main occupation of respondents was agriculture as this sector alone was practiced by $94.5 \%$. This is in line with the figures from the National Institute of Statistics for Rwanda (NISR) 2015 report which also revealed similar statistics for employment occupation in rural Rwanda.

Table 1. Demographic information of participants

\begin{tabular}{lr}
\hline Variables & $\mathbf{N ( \% )}$ \\
\hline Sex & \\
Male & $206(70.8 \%)$ \\
Female & $85(29.2 \%)$ \\
Total & $\mathbf{2 9 1 ( 1 0 0 )}$ \\
\hline Occupations & \\
Agricultural & $275(94.5 \%)$ \\
Day laborer & $4(1.4 \%)$ \\
Service/ Sales / Commercial & $3(1 \%)$ \\
Professional /Technical & $4(1.4 \%)$ \\
Pottery & $1(0.3 \%)$ \\
No occupation & $4(1.4 \%)$ \\
Total & $\mathbf{2 9 1 ( 1 0 0 )}$ \\
\hline
\end{tabular}

\section{Hand washing with soap (HWWS)}

The findings revealed that one-third of all participants used to wash hands with soap and water after cleaning child bottom and $34.4 \%$ wash hands with soap and water after touching any dirt.
Hand washing before breastfeeding was only among $0.3 \%$ as a participant even pinpointed during FGDs that "mothers rarely wash their hands before breastfeeding". 
The findings have shown that $85.9 \%$ of respondents have clear knowledge on ideal practices on hand washing with soap but on the other hand, washing hands using water only was a common practice across all study locations (villages). Washing hands with water alone could not be effective in leaving them free of infections.[12] According to the discussions, it has also emerged when participants quoted that they "rarely use soap during hand washing". Most of the respondents agreed that soaps should be present at the place of hand washing but because of poverty soap seems to be expensive; they were not able to use it.[12]

Table 2. Hand washing status at critical time

\begin{tabular}{lr}
\hline Hand washing critical time & N (\%) \\
\hline Hand washing with soap at critical times is not common in my community & $41(14.1 \%)$ \\
Hand washing with soap after defecation is common in the community & $160(55 \%)$ \\
Hand washing with soap before feeding/eating are common in the community & $226(77.7 \%)$ \\
Hand washing with soap and water after cleaning child bottom & $96(33 \%)$ \\
Hand washing with soap and water after touching any dirt / dust/waste & $100(34.4 \%)$ \\
Hand washing with soap and water before breastfeeding & $1(0.3 \%)$ \\
Don't know & $1(0.3 \%)$ \\
\hline
\end{tabular}

\section{Critical moments for hand washing}

Hand washing with water and soap before eating and before feeding children was reported among $36.2 \%$ of the study participants. More than half of participants $(55 \%)$ reported washing hand with soap after defecation and a small proportion of $6.6 \%$ reported not to practice hand washing at any hand washing critical moment. Similar findings have been highlighted in the literature where hand washing using water and soap after defecation has been reported nearly among half of the participants. [13]

It was observed that most of participants lack hand washing facilities. To add on this, it has emerged during FDGs that some participants have shortage of water and may not always be able to buy and use soaps. A large proportion (95\%) of the respondents perceived that hand washing can sustain children's future. It was observed that hand washing is practiced before and after eating, before milking a cow, and preparing food. It was also practiced after cleaning the compound, cooking, using toilet, and touching and disposing waste. However, participants may decide at which moment to use or not use soap, in the FGDs participants said that "people prioritize use of soap in other hygienic practices including laundry, washing dishes and bathing". This statement informs about the insufficiency of soap and its rational use may lead to limited frequency.[12, 14]

This is in line with the findings from the report from NISR where low hygiene practices were reported as only 12 per cent of households had a place for hand washing. Moreover, hand washing practice increases with increasing wealth.[7] Other research reports showed that $59.4 \%$ of the study households use water and soap for cleaning dirty hands and $37.4 \%$ of the household members of the study participants use water and soap after defecation. The same study revealed that cleaning hands with water before and after meals was highly practiced.[15]

\section{Faecal management}

The findings revealed that nearly all respondents own pit latrines and of which $65 \%$ use unimproved latrines with single offset pit toilets and a few were direct pit toilets with just-reinforced walls and in most cases without doors. Additionally, the toilets constructed do not have a roof against rain; the superstructure does not provide sufficient privacy for the users. They intended to improve the roof, the slab, and building hand washing facilities. The participants ideally believed that an ideal latrine should be clean and without bad smell. The study revealed that $96 \%$ of respondents drop child faeces into the toilet, while a small proportion of participants stated that they dispose child faeces into drain or ditch, throw into garbage, or bury it into the field.[14] The findings from FGDs have been very surprising where in all discussions, participants have pointed out that "some people still practice open defecation to be able to feed their livestock especially pigs" while others have revealed that "due to lack of fertilisers, some farmers choose to defecate in their fields (cultivating land) for 
fertilization" however the country registers a small percentage of open defecation while this may lead to number of fatal consequences.[16, 17]

Table 3. Respondents' intended toilet improvements

\begin{tabular}{lr}
\hline Variables & $\mathbf{N ~ ( \% )}$ \\
\hline Toilet improvement & \\
Improve the Slab & $80(27.5 \%)$ \\
Improve the Roof & $119(40.9 \%)$ \\
Line the pit & $28(9.6 \%)$ \\
Move to inside the house & $7(2.4 \%)$ \\
Build hand washing area & $6(2.1 \%)$ \\
Build bathing area & $57(19.6 \%)$ \\
Get pan & $7(2.4 \%)$ \\
Get pour flush pan & $1(0.3 \%)$ \\
Build door & $1(0.3 \%)$ \\
Build a new one & $4(1.4 \%)$ \\
Add ventilation pipe to pit & $2(0.7 \%)$ \\
\hline Child faeces disposal & \\
\hline In the toilet & $285(97.9 \%)$ \\
Put into drain or ditch & $2(0.7 \%)$ \\
Throw into garbage & $1(0.3 \%)$ \\
In fields & $1(0.3 \%)$ \\
No child & $1(0.3 \%)$ \\
Bury & $2(0.7 \%)$ \\
Given to animals (pigs) & $1(0.3 \%)$ \\
\hline
\end{tabular}

\section{Household water treatment}

The findings from this survey showed that more than a half $55 \%$ of the participants do not treat water while $44 \%$ use boiling methods. Only a very small proportion of participants $(1 \%)$ use chlorination. Participants in FGDS have claimed that "the lack of firewood and water treatment products in the community has been pinpointed as the main challenge to treating water". In other studies, household water treatment and safe storage (HWTS), such as boiling or chlorinating water at home, have been shown to be effective in improving the quality of drinking water. $[18,19]$

This requires interventions driven into household water treatment to improve the quality of drinkingwater and reduce possible diarrhoeal disease. 


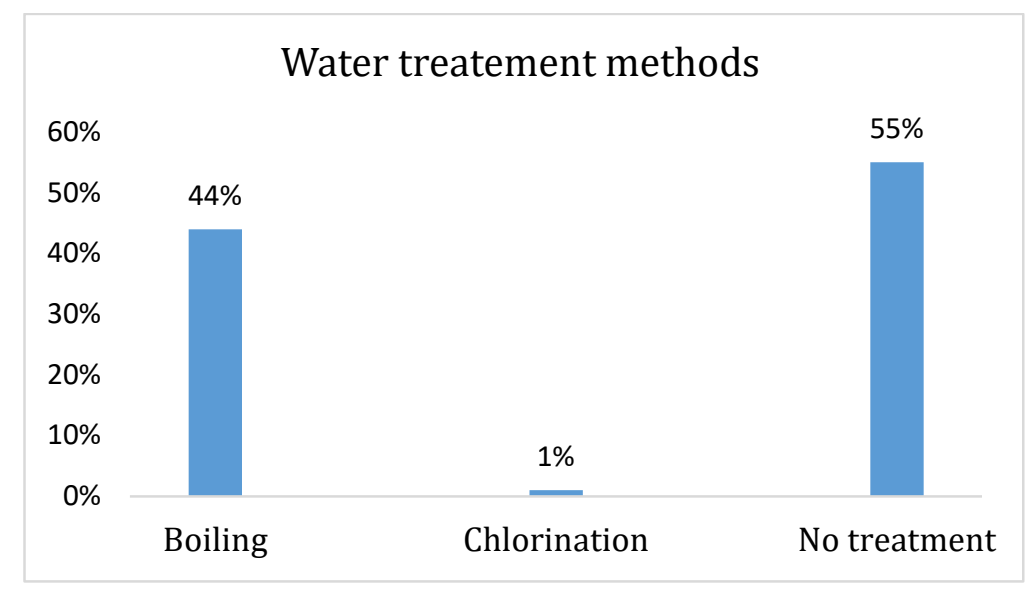

\section{Figure 1. Water treatment methods}

\section{Household water storage}

The findings showed that a relative high proportion of respondents use clean and covered containers as it was reported by $61 \%$ of households who use plastic jerrycans covered with clean lid. $15.5 \%$ of the households reported that they keep water in clean utensils. However, $21.3 \%$ of households do not store water. The latter may be exposed to some waterborne diseases especially those who use unprotected water sources. It is therefore important to carry out interventions to improve the quality of drinking water, particularly among those who rely on water from unimproved sources, and in some cases, unsafe or unreliable piped water supplies. The water storage is also very critical as studies show that its condition may link to re-growing of microbes in water potable supplies, [20] this may be caused by some water related characteristics including temperature as significant bacteria may grow in water at $\geq 15^{\circ} \mathrm{C}$, water turbidity as the water suspension matters like clay, silt, organic and inorganic matters and microorganisms may either provide nutrients to bacteria and pathogens or protect them from chlorination.[21]

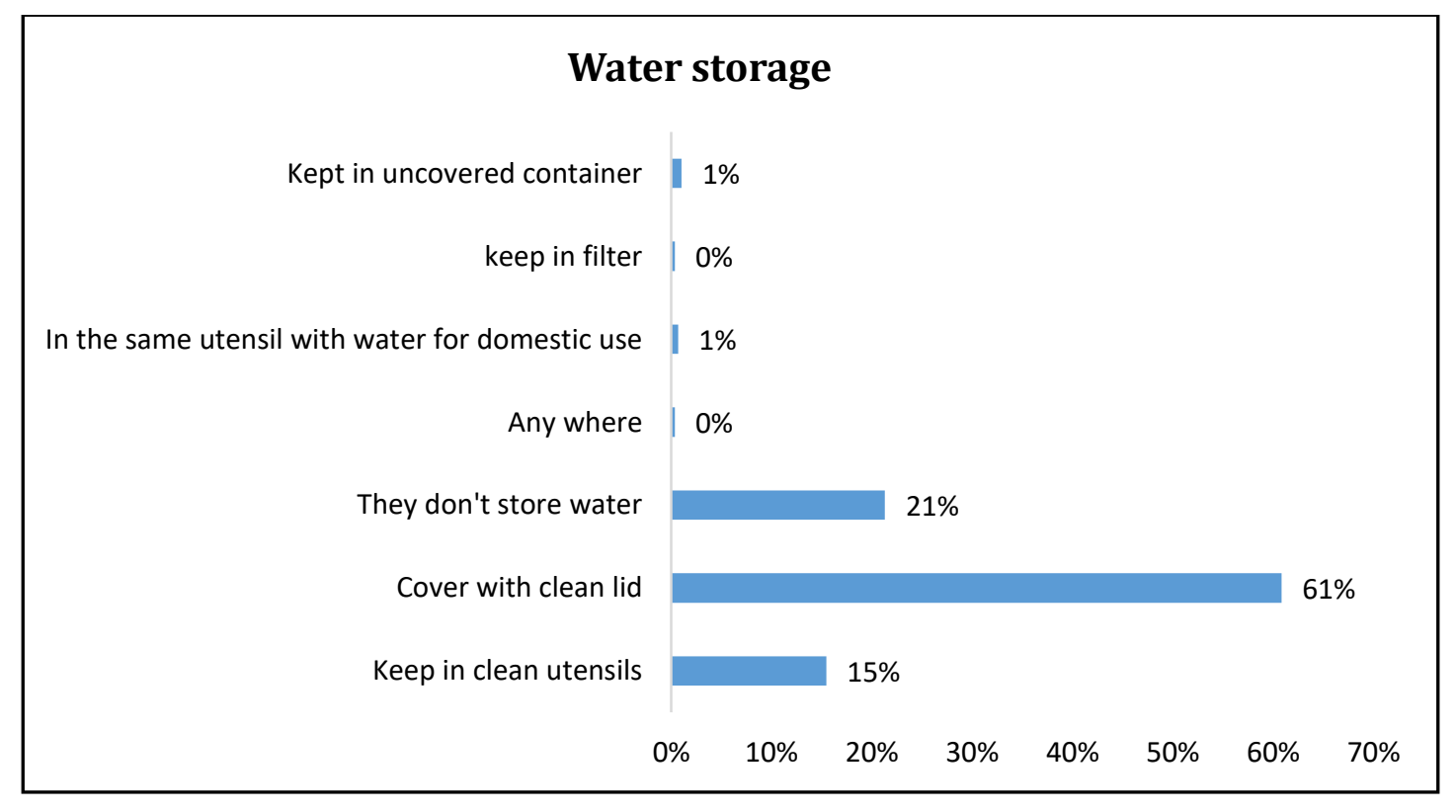

Figure 2. Household water storage methods 
The household treatment of water is essential in increasing water quality and reducing exposure to diarrheal diseases. There are five major options most widely used; they include chlorination, disinfectant powder also known as flocculants, solar disinfection, ceramic filtration and slow sand filtration.[22, 23] One of the challenge is also the factors related to selection of the method in use, this would depend on existing water and sanitation conditions, water quality, cultural acceptability, implementation feasibility, availability of technology, and other local conditions.[11, 22]

The findings from the present study revealed that only a small portion $0.4 \%$ of participants don't know if drinking untreated water can cause diseases, but the rest of participants at least know one disease caused by untreated water. Participants had a perception that drinking untreated water can be the source of different diseases. Some diseases that were perceived to be the outcome of untreated water intake included $65.3 \%, 48.1 \%$, and $29.6 \%$ worm diseases, diarrhoea, and cholera respectively.

\section{Food hygiene practices}

Cleaning hands before preparing food for consumption was a common practice among the participants, where by $96 \%$ of respondents indicated that they ensure their hands are cleaned before or while preparing food. When investigating this dimension, only $3.4 \%$ of respondents stated that they apply different techniques to wash raw food before consumption; this explains that the majority did not use running water, this presents a risk of re-contamination of the food. Most used a container such as a basin or bucket, filled with water to soak and scrub the food in the water.

One informant said, "People in this community don't use running water when it comes to wash raw food. Usually they wash it in one container, then re-wash it again." Only a few used running water or poured water. Also, the household survey showed that in general, they do it to remove dirt that is considered to be a source of disease.[24] During the discussions there was a fairly strong negative perception of people who did not wash food before preparing it. It was also found that the participants have certain guidelines or criteria to determine whether a particular kind of food needs to be washed or not. With regard to the actual washing, the study found that some participants used techniques that could re-contaminate the food[25], this calls upon the accessibility to knowledge and behaviour change modalities.

\section{CONCLUSION}

The survey provided behavioural factors associated with hand washing with soap and food hygiene practices. Lack of water and soaps, and hand washing facilities were among other factors that hinder hygiene and sanitation. Insufficient public toilets facilities along with charging fees were found to be key factor that favor defecation in the open in some public places though this is not common. The local community used boiling water as their main water treatment methods but this is affected by the lack of firewood and as a result people end up drinking untreated water which causes a widespread of waterborne diseases like diarrhea.

\section{RECOMMENDATIONS}

Based on the finding, we recommend the Local authorities (District authorities) to set messages to convey key information regarding hand washing with soap and to assist private sector federation members who are willing to invest in sanitation material and services. There is need to strengthen hygiene and sanitation collaboration by building bridges between various institutions involved. We also recommend collaboration of the district authority and stakeholders in dissemination of relevant information through community health clubs. Community health clubs must be the subject of special attention during any hygiene and sanitation intervention. In addition, parents and guardians need to have a sense of ownership and adopt correct hygiene practice.

\section{Acknowledgements}

We acknowledge WaterAid Rwanda for providing funds for the research. Special thanks to all contributors including Nyamagabe District authorities.

\section{Authors' contribution}

TN and MT both contributed to all steps of the work from the design, data collection, data analysis and manuscript write-up. SFX and ON contributed to the manuscript revision

\section{Conflict of interest}

Authors declare that there is no conflict of interest.

This article is published open access under the Creative Commons Attribution-NonCommercialNoDerivatives (CC BYNC-ND4.0). People can copy and redistribute the article only for non commercial purposes and as long as they give appropriate credit to the authors. They cannot distribute any modified material obtained by remixing, 
transforming or building upon this article. See

https://creativecommons.org/licenses/by-nc-nd/4.0/

\section{REFERENCES}

1. Kuberan A, Singh A, Kasav J, Prasad S, Surapaneni KM, Upadhyay V, Joshi A (2015) Water and sanitation hygiene knowledge, attitude, and practices among household members living in rural setting of India. J Nat Sci Biol Med 6:S69-S74

2. Guo A, Bowling JM, Bartram J, Kayser G (2017) Water, sanitation, and hygiene in rural health-care facilities: a cross-sectional study in Ethiopia, Kenya, Mozambique, Rwanda, Uganda, and Zambia. Am J Trop Med Hyg 97:1033-1042

3. Zhou X, Li Z, Zheng T, Yan Y, Li P, Odey EA, Mang HP, Uddin SMN (2018) Review of global sanitation development. Environ Int 120:246-261

4. Government of Rwanda (2019) 2019 Rwanda Voluntary National Review (VNR) Report. 1109

5. MININFRA (2017) Achieving the SDGs targets for water, sanitation and hygiene Rwanda Overview: Water, Sanitation and Hygiene.

6. Republic of Rwanda Ministry of Infrastructure (2010) National Policy \& Strategy for Water Supply and Sanitation Services.

7. Kamayirese G (2016) Republic of Rwanda Ministry of Infrastructure National Sanitation Policy.

8. Tsinda A, Abbott P (2018) Critical Water, Sanitation and Hygiene (WASH) Challenges in Rwanda.

9. WHO (2014) Exposures and impacts in lowand middle-income countries preventing diarrhoea through better water, sanitation and hygiene.

10. NISR (2017) EICV 5 - Rwanda Poverty Profile Report 2016/17 | National Institute of Statistics Rwanda.

11. Hasselskog M (2016) Participation or What? Local Experiences and Perceptions of Household Performance Contracting in Rwanda. Forum Dev Stud 43:177-199
12. Luby SP, Halder AK, Huda T, Unicomb L, Johnston RB (2011) The effect of handwashing at recommended times with water alone and with soap on child diarrhea in rural Bangladesh: An observational study. PLoS Med. doi: 10.1371/journal.pmed. 1001052

13. Reddy VB, Kusuma YS, Pandav CS, Goswami AK, Krishnan A (2017) Water and Sanitation Hygiene Practices for Under-Five Children among Households of Sugali Tribe of Chittoor District, Andhra Pradesh, India. J Environ Public Health. doi: 10.1155/2017/7517414

14. NISR (2015) Rwanda Demographic and Health Survey.

15. Reddy B V, Kusuma YS, Pandav CS, Kumar Goswami A, Krishnan A (2017) Water and Sanitation Hygiene Practices for Under-Five Children among Households of Sugali Tribe of Chittoor District, Andhra Pradesh, India. doi: $10.1155 / 2017 / 7517414$

16. Coffey D, Gupta A, Hathi P, Khurana N, Spears D, Srivastav N, Vyas S (2014) Revealed Preference for Open Defecation. Econ Polit Wkly 49:43-55

17. Coffey D, Gupta A, Hathi P, Spears D, Srivastav N, Vyas S (2017) Understanding open defecation in rural India untouchability, pollution, and latrine pits. Econ Polit Wkly 52:59-66

18. Stubbé SML, Pelgrim-Adams A, Szántó GL, van Halem D (2016) Household water treatment and safe storage - effectiveness and economics. Drink Water Eng Sci 9:9-18

19. Clasen T (2015) Household Water Treatment and Safe Storage to Prevent Diarrheal Disease in Developing Countries. Curr Environ Heal reports 2:69-74

20. VanDerslice JPG and J (2007) The effectiveness of large household water storage tanks for protecting the quality of drinking water. J. Water Health

21. Schafer CA (2010) Impact of Tank Material on water Quality in Household Water Storage Systems in Cochabamba, Bolivia. 130

22. Ma S, Gan Y, Chen B, Tang Z, Krasner S (2017) Understanding and exploring the potentials of household water treatment methods for volatile disinfection by-products 
Rwanda Journal of Medicine and Health Sciences Vol.3 No.1, March 2020 https://dx.doi.org/10.4314/rjmhs.v3i1.6

control: Kinetics, mechanisms, and influencing factors. J Hazard Mater 321:509516

23. Lantagne DS, Clasen TF (2012) Use of household water treatment and safe storage methods in acute emergency response: Case study results from Nepal, Indonesia, Kenya, and Haiti. Environ Sci Technol 46:1135211360
24. Aigbiremolen $\mathrm{AO}$, Abejegah $\mathrm{C}$, Ike $\mathrm{CG}$, Momoh JA, Abah SO (2015) Knowledge and practice of hand washing among caregivers of under-five children in a rural Nigerian community. Public Heal Res 5:159-165

25. Soon JM, Baines R, Seaman P (2012) Metaanalysis of food safety training on hand hygiene knowledge and attitudes among food handlers. J Food Prot 75:793-804 